\title{
Adaptive-search template matching technique based on vehicle acceleration for monocular visual odometry system
}

\begin{abstract}
One of the important tasks of an autonomous mobile vehicle is the reliable and fast estimation of its position over time. This paper presents the development of an adaptive technique to hasten and improve the quality of correlation-based template matching for monocular visual odometry systems that estimate the relative motion of ground vehicles in low-textured environments. Moreover, the factors that can affect the maximum permissible vehicle driving speed were determined and the related equations were derived. The developed system uses a single downward-facing monocular camera installed at an optimum location to avoid the negative effect of directional sunlight and shadows which can disturb the correlation. In addition, the normalized cross-correlation method is implemented to calculate the pixel displacement between image frames. Although this method is highly effective for template matching because of its invariance to linear brightness and contrast variations, it incurs high computational cost. Thus, the optimal sizes of image template and matching search area are selected and their locations are dynamically changed according to vehicle acceleration, in order to achieve a compromise between the performance and the computational cost of correlation. The proposed technique increases the allowable vehicle driving speed and reduces the probability of template false-matching. Moreover, compared to traditional full search matching techniques, the adaptive technique demonstrates high efficiency and accuracy and improves the quality and speed of the correlation with more than $87 \%$ of reduction in computational cost.
\end{abstract}

Keyword: Monocular visual odometry; Downward-facing camera; Adaptive search; Template-matching; Low-textured environments; Motion estimation 